FR-PHENO-2010-021

\title{
NLO QCD corrections to 4 b-quark production
}

\author{
Nicolas Greiner* \\ University of Illinois at Urbana-Champaign, Urbana IL, 61801 USA \\ E-mail: ngreiner@illinois.edu
}

\section{Alberto Guffanti}

Physikalisches Institut, Albert-Ludwigs-Universität Freiburg, 79104 Freiburg i. Br., Germany

E-mail: alberto.guffanti@physik.uni-freiburg.de

\section{Jean-Philipe Guillet}

LAPTH, Annecy-le-Vieux 74951, France

E-mail: guilletelapp.in2p3.fr

\section{Thomas Reiter}

Nikhef, 1098XG Amsterdam, The Netherlands

E-mail: thomasr@nikhef.nl

\section{Jürgen Reuter}

Physikalisches Institut, Albert-Ludwigs-Universität Freiburg, 79104 Freiburg i. Br, Germany

E-mail: juergen.reuter@physik.uni-freiburg.de

In this talk we present the recent calculation of the NLO QCD corrections to the production of four b-quarks induced by initial state quarks at the CERN LHC. We describe the details of the calculation using the GOLEM approach for the virtual corrections and MadEvent/MadDipole for the real emission part and present some results.

XVIII International Workshop on Deep-Inelastic Scattering and Related Subjects, DIS 2010 April 19-23, 2010

Firenze, Italy

\footnotetext{
*Speaker.
} 


\section{Introduction}

The study of the Higgs sector of the Standard Model (SM) and the search for New Physics at the $\mathrm{TeV}$ scale at the LHC experiments at CERN will ultimately depend on a detailed understanding of signal and background processes involving many particles [1, 2, 3, 4].

When considering hadronic collisions the results obtained using only leading order (LO) in perturbation theory retain a strong dependence on the unphysical factorisation and renormalisation scales which limits the predictivity of the theoretical computations.

Whereas the inclusion of next-to-leading order (NLO) QCD effects is relatively straightforward for simple kinematic situations ( $2 \rightarrow 2$ and $2 \rightarrow 3$ processes), processes with four parton final states are at the forefront of the present computing capabilities and the number of available results is very limited. However a lot of progress has been made during the last couple of years. Recent achievements are the evaluation of the order $\alpha_{s}$ corrections to $p p \rightarrow b \bar{b} t \bar{t}[5,6,7], p p \rightarrow W j j j$ $[8,9,10,11], p p \rightarrow t \bar{t} j j[12]$ and $p p \rightarrow Z / \gamma j j j[13]$.

In this contribution we present the results obtained in [14] for the process $p p \rightarrow b \bar{b} b \bar{b}$ which is a relevant background for Higgs searches in the minimal supersymmetric extension of the Standard Model (MSSM). The MSSM contains two Higgs doublets containing five scalar Higgs bosons. As light Higgs bosons prefer to decay into $b$-quarks in large parts of parameter space, experimental studies are so far strongly affected by the uncertainty of the Standard Model backgrounds, especially $p p \rightarrow b \bar{b} b \bar{b}$, see for example Ref. [15]. Although mainly motivated by supersymmetry, the four- $b$ final state also allows the study of other interesting scenarios for Beyond Standard Model (BSM) physics, such as Hidden Valley models, where decays of hadrons of an additional confining gauge group can produce high multiplicities of $b \bar{b}$ pairs $[2,16]$. This is the reason why the $p p \rightarrow b \bar{b} b \bar{b}$ process was added to the experimenters wishlist of relevant next-to-leading order computations [2].

\section{Calculation}

At leading order two partonic subprocesses contribute to the computation of the $p p \rightarrow b \bar{b} b \bar{b}$ cross-section: the quark initiated subprocess $(q \bar{q} \rightarrow b \bar{b} b \bar{b})$ and the gluon initiated one $(g g \rightarrow b \bar{b} b \bar{b})$. In this contribution we presents results obtained for the quark induced subprocess Throughout the whole calculation we treat the $b$-quarks as massless, an approximation which is well motivated by the LHC kinematics and the applied cuts, and neglect the effect of a heavy top quark altogether. Furthermore we neglect the $b$-quark contribution in the parton distribution functions, due to the smallness of the $b$ PDF.

\subsection{Virtual corrections}

The calculation of the virtual corrections is based on the GOLEM approach [17, 18, 19, 21] for one loop amplitudes. It uses a Feynman diagrammatic representation generated by QGRAF [23]. FORM [24] is used to map the helicity amplitudes to a tensor form factor representation as defined in $[17,22,25]$. The latter is exported to Fortran 95 code such that it can be linked to the form factor library golem 95 [20]. For the code generation we have developed a dedicated optimisation tool which can also be used for other purposes [26]. To validate the results a second independent 
calculation has been performed. There we did a symbolic tensor reduction down to genuine scalar integrals where we used FeynArts and FeynCalc [27] to generate the amplitudes. The tensor integrals are reduced symbolically to scalar integrals also using the formalism described in [17].

More recently we have independently calculated the virtual corrections using the SAMURAI package [38]. SAMURAI is a package for the reduction of one-loop amplitudes at the integrand level based on the OPP approach [39, 40]. Combined with a modified version of our matrix element generator the code for the numeric evaluation of the matrix element was generated in a fully automated way.

Using this setup we were able to reduce the CPU time needed for the evaluation of the virtual corrections of a single phase space point to around $0.4 \mathrm{~s}$, which is around a factor of 10 faster compared to the previous approach.

This improvement in the code speed is a fundamental step towards the computation of the gluon induced subprocess which, due to the larger number of Feynman diagrams and their increased complexity, is computationally more intensive than the quark initiated one.

\subsection{Real emission}

The tree level and real emission matrix elements have been generated using MadGraph [28], for the subtraction terms we used MadDipole [29, 30] which is based on the dipole formalism $[31,32]$. For the full proton initiated process there are three subprocesses contributing: $q \bar{q} \rightarrow$ $b \bar{b} b \bar{b} g, g g \rightarrow b \bar{b} b \bar{b} g$ and $q g \rightarrow b \bar{b} b \bar{b} q$. Here we restrict ourselves to the first one. The subtraction terms and the real emission matrix element have been implemented into the MadEvent framework [33].

A second implementation of the subtraction terms in Whizard $[34,35]$ has been used to ensure the correctness of our code.

\section{Results}

In order to provide a check of the numerical stability of our implementation we looked at the cancellation of the double and single poles between the virtual corrections and dipole subtraction terms. The result is presented in Fig. 1 and shows that the cancellation happens within double precision accuracy for both single and double poles, thus confirming that possible numerical instabilities are well under control.

The numerical results we present in this section were obtained for $\sqrt{s}=14 \mathrm{TeV}$ center of mass energy. In a first step we cluster the final state particles to $b$-jets using the $k_{T}$-algorithm [36]. The resulting jets have to pass the following cuts:

$$
\begin{aligned}
p_{T}\left(b_{j}\right) & >30 \mathrm{GeV} \\
\left|\eta\left(b_{j}\right)\right| & <2.5 \\
\Delta R\left(b_{i}, b_{j}\right) & >0.8 .
\end{aligned}
$$

One of the main goals of calculating higher orders corrections is to reduce the dependence of the result on renormalisation and factorisation scale. In Fig. 2 we compare the dependence on the renormalisation scale for the total cross section and for the invariant mass distribution of the two 


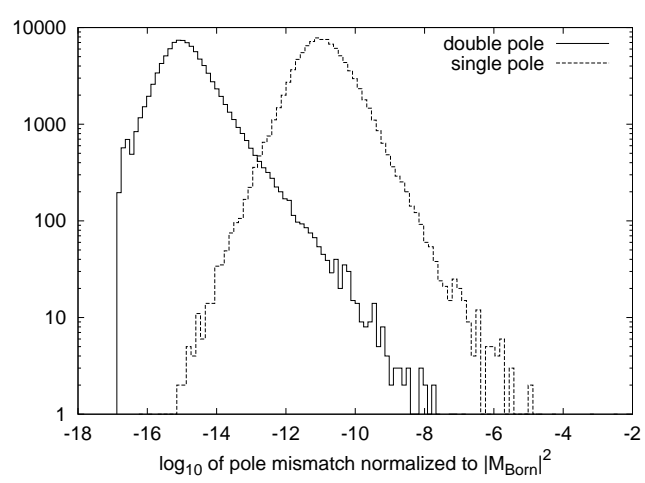

Figure 1: Cancellation of the poles from the virtual corrections and the subtraction terms normalised to the Born. To produce this plot we integrated the process $q \bar{q} \rightarrow b \bar{b} b \bar{b}$ using $10^{5}$ phase space points and the cuts described in the text.
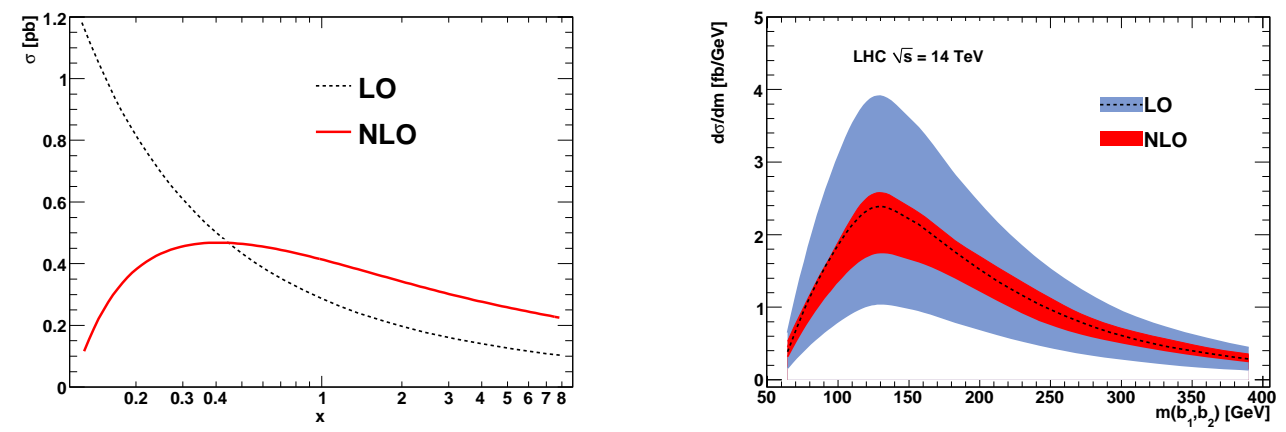

Figure 2: Dependence on the renormalisation scale for the total cross section (left plot) and for the distribution of the invariant mass of the two $b$-jets with the higest $p_{T}$ (right plot) when varying the scale around $\mu_{r}=x \cdot \mu_{0}=x \cdot \sqrt{\sum_{j} p_{T}^{2}\left(b_{j}\right)}$ with $x \in\left[\frac{1}{8}, 8\right]$ (left plot) and $x \in\left[\frac{1}{4}, 2\right]$. (right plot). The dashed line in the right plot denotes the leading order prediction for $x=1 / 2$.

$b$-jets with the highest $p_{T}$ at LO and NLO. As expected, a clear reduction on the scale dependence can be observed. The central scale is chosen to be $\mu_{0}=\sqrt{\sum_{j} p_{T}^{2}\left(b_{j}\right)}$ and the scale is then varied with $\mu_{r}=x \cdot \mu_{0}$. As we have not included all possible initial states we kept the factorisation scale fixed to be $\mu_{F}=100 \mathrm{GeV}$.

\section{Acknowledgments}

N.G. is supported by the U. S. Department of Energy under contract No. DE-FG02-91ER40677. T.R. has been supported by the Foundation FOM, project FORM 07PR2556.

\section{References}

[1] N. E. Adam et al., arXiv:0803.1154 [hep-ph].

[2] Z. Bern et al. [NLO Multileg Working Group], arXiv:0803.0494 [hep-ph]. 
[3] C. Buttar et al., hep-ph/0604120.

[4] J. M. Campbell, J. W. Huston and W. J. Stirling, Rept. Prog. Phys. 70 (2007) 89 [hep-ph/0611148].

[5] A. Bredenstein, A. Denner, S. Dittmaier and S. Pozzorini, JHEP 0808, 108 (2008) [arXiv:0807.1248 [hep-ph]].

[6] A. Bredenstein, A. Denner, S. Dittmaier and S. Pozzorini, Phys. Rev. Lett. 103, 012002 (2009) [arXiv:0905.0110 [hep-ph]].

[7] G. Bevilacqua, M. Czakon, C. G. Papadopoulos, R. Pittau and M. Worek, JHEP 0909, 109 (2009) [arXiv:0907.4723 [hep-ph]].

[8] C. F. Berger et al., Phys. Rev. D D80, 074036 (2009) [arXiv:0907.1984 [hep-ph]].

[9] C. F. Berger et al., Phys. Rev. Lett. 102, 222001 (2009) [arXiv:0902.2760 [hep-ph]].

[10] R. K. Ellis, K. Melnikov and G. Zanderighi, arXiv:0906.1445 [hep-ph].

[11] R. K. Ellis, K. Melnikov and G. Zanderighi, JHEP 0904 (2009) 077 [arXiv:0901.4101 [hep-ph]].

[12] G. Bevilacqua, M. Czakon, C. G. Papadopoulos and M. Worek, Phys. Rev. Lett. 104, 162002 (2010) [arXiv:1002.4009 [hep-ph]].

[13] C. F. Berger et al., arXiv:1004.1659 [hep-ph].

[14] T. Binoth, N. Greiner, A. Guffanti, J. Reuter, J. P. Guillet and T. Reiter, Phys. Lett. B 685 (2010) 293 [arXiv:0910.4379 [hep-ph]].

[15] R. Lafaye, D. J. Miller, M. Muhlleitner and S. Moretti, hep-ph/0002238.

[16] W. Krolikowski, arXiv:0803.2977 [hep-ph].

[17] T. Binoth, J. P. Guillet, G. Heinrich, E. Pilon and C. Schubert, JHEP 0510 (2005) 015 [hep-ph/0504267].

[18] T. Binoth et al., arXiv:0807.0605 [hep-ph].

[19] T. Binoth, J. P. Guillet and G. Heinrich, Nucl. Phys. B 572 (2000) 361 [hep-ph/9911342].

[20] T. Binoth, J. P. Guillet, G. Heinrich, E. Pilon and T. Reiter, Comput. Phys. Commun. 180, 2317 (2009) [arXiv:0810.0992 [hep-ph]].

[21] T. Binoth, J. P. Guillet and G. Heinrich, JHEP 0702, 013 (2007) [hep-ph/0609054].

[22] T. Reiter, arXiv:0903.0947 [hep-ph].

[23] P. Nogueira, J. Comput. Phys. 105, 279 (1993).

[24] J. A. M. Vermaseren, arXiv:math-ph/0010025.

[25] T. Reiter, arXiv:0903.4648 [hep-ph].

[26] T. Reiter, arXiv:0907.3714 [hep-ph].

[27] T. Hahn and M. Perez-Victoria, Comput. Phys. Commun. 118, 153 (1999) [hep-ph/9807565].

[28] T. Stelzer and W. F. Long, Comput. Phys. Commun. 81, 357 (1994) [hep-ph/9401258].

[29] R. Frederix, T. Gehrmann and N. Greiner, JHEP 0809 (2008) 122 [arXiv:0808.2128 [hep-ph]].

[30] R. Frederix, T. Gehrmann and N. Greiner, arXiv:1004.2905 [hep-ph]. 
[31] S. Catani and M. H. Seymour, Nucl. Phys. B 485, 291 (1997) [Erratum-ibid. B 510, 503 (1998)] [hep-ph/9605323].

[32] S. Catani, S. Dittmaier, M. H. Seymour and Z. Trocsanyi, Nucl. Phys. B 627, 189 (2002) [hep-ph/0201036].

[33] F. Maltoni and T. Stelzer, JHEP 0302, 027 (2003) [hep-ph/0208156].

[34] W. Kilian, T. Ohl and J. Reuter, arXiv:0708.4233 [hep-ph].

[35] M. Moretti, T. Ohl and J. Reuter, arXiv:hep-ph/0102195.

[36] G. C. Blazey et al., hep-ex/0005012.

[37] A. Cafarella, C. G. Papadopoulos and M. Worek, Comput. Phys. Commun. 180, 1941 (2009) [arXiv:0710.2427 [hep-ph]].

[38] P. Mastrolia, G. Ossola, T. Reiter and F. Tramontano, arXiv:1006.0710 [hep-ph].

[39] G. Ossola, C. G. Papadopoulos and R. Pittau, Nucl. Phys. B 763, 147 (2007) [hep-ph/0609007].

[40] G. Ossola, C. G. Papadopoulos and R. Pittau, JHEP 0707, 085 (2007) [arXiv:0704.1271 [hep-ph]]. 